\title{
5G and EMF Exposure: Misinformation, Open Questions, and Potential Solutions
}

\author{
Ahmed Elzanaty $^{1 *}$, Luca Chiaraviglio ${ }^{2,3}$ and Mohamed-Slim Alouini ${ }^{1}$ \\ ${ }^{1}$ Computer, Electrical, and Mathematical Science and Engineering Division, King Abdullah University of Science and Technology \\ (KAUST), Thuwal, Saudi Arabia, ${ }^{2} E$ lectrical Engineering Department, University of Rome Tor Vergata, Rome, Italy, ${ }^{3}$ Consorzio \\ Nazionale Interuniversitario per le Telecomunicazioni, Parma, Italy
}

The massive deployment of advanced wireless networks is essential to support broadband connectivity, low latency communication, and Internet of Things applications. Nevertheless, in the time of coronavirus disease (COVID-19) there is a massive amount of misinformation and uncertainty about the impact of fifth-generation cellular network (5G) networks on human health. In this paper, we investigate the main categories of misinformation regarding $5 \mathrm{G}$, i.e., fake theories, the misconception of $5 \mathrm{G}$ features, and open questions that require further research. Then, we propose two novel approaches for the design of electromagnetic field (EMF)-aware cellular networks that can reduce human exposure to radio frequency radiation.

Edited by:

Nidal Nasser,

Alfaisal University, Saudi Arabia

Reviewed by:

Nathalie Mitton,

Institut National de Recherche en Informatique et en Automatique,

France

Luca Bedogni,

University of Modena and Reggio

Emilia, Modena, italy

*Correspondence:

Ahmed Elzanaty

ahmed.elzanaty@kaust.edu.sa

$$
\begin{array}{r}
\text { Specialty section: } \\
\text { This article was submitted to } \\
\text { Networks, } \\
\text { a section of the journal } \\
\text { Frontiers in Communications } \\
\text { and Networks }
\end{array}
$$

Received: 30 November 2020

Accepted: 15 February 2021

Published: 19 April 2021

Citation:

Elzanaty A., Chiaraviglio L. and Alouini M.-S. (2021) 5G and EMF

Exposure: Misinformation, Open

Questions, and Potential Solutions.

Front. Comms. Net 2:635716.

doi: 10.3389/frcmn.2021.635716
Keywords: EMF (electromagnetic field), $5 \mathrm{G}$ networks and beyond, radiation adverse effects, UAV (unmanned aerial vehicle), EMF-aware cellular systems, COVID-19

\section{INTRODUCTION}

The fifth-generation cellular network $(5 \mathrm{G})$ is endorsed by its support of high data rate communications for an increasing number of users. One solution to approach the key performance indicators of $5 \mathrm{G}$ is to adopt millimeter-waves (mm-waves) and install a massive number of base stations (BSs) and small cells, i.e., network densification. Recently, a large portion of the population is concerned about potential health impacts from being exposed to $5 \mathrm{G}$ radio frequency radiation (RFR) (Nyberg and Hardel, 2017). The arisen doubts about 5G can delay the deployment of 5G in some countries, leading to unfavorable economic impacts (Jones, 2020).

RFR is non-ionizing radiation that involves waves with smaller energy that cannot ionize the cells, thus preventing severe impacts on the exposed cells. Nevertheless, the electromagnetic field (EMF) can cause the molecules to vibrate, leading to a possible heating effect on the exposed tissues, i.e., thermal effect (Foster et al., 2017). In this regard, regulatory authorities such as International Commission on Non-Ionizing Radiation Protection (ICNIRP) and Federal Communications Commission (FCC) quantify the maximum allowable radio frequency (RF) exposure to limit the temperature rising of the exposed tissues (Robert Cleveland and Sylvar, 1997; ICNIRP, 1998; IEEE, 2005; ICNIRP, 2020). Regarding the non-thermal long-term exposure to $5 \mathrm{G}$, there is a debate in the community about whether it has adverse health impacts. However, no adverse effects have been proven for exposure that is coherent with the regulatory limits, as shown in Section 2.3.1.

The doubt about 5G is induced by fake theories and hoaxes that are not based on any scientific evidence. Also, the misconception of $5 \mathrm{G}$ operational principles and the open questions, always associated with new technologies, can drive the population to overly perceive the health risks from 
$5 \mathrm{G}$ exposure. One of these fake theories attribute coronavirus disease (COVID-19) spread to the EMF exposure from 5G. The massive number of hoaxes trigger the World Health Organization (WHO) to use the expression "infodemic" to describe the vast amount of misinformation regarding Covid19, which can have severe impacts on the community (WHO, 2020a). For example, several sabotage actions have been committed toward 5G towers in some countries (BBC, 2020; Liverpoolecho, 2020). In the next section, we investigate some arguments that drive the fair from $5 \mathrm{G}$ networks.

\section{MISINFORMATION AND OPEN QUESTIONS}

In this section, we discuss the misinformation regarding 5G, which can be classified into two main groups: 1) fake theories and hoaxes and 2) misconception of 5G features. Also, some open questions requiring further research are investigated.

\subsection{Fake Theories and Hoaxes}

Many fake and conspiracy theories have been circulating in the media regarding alleged adverse health impacts due to the EMF exposure from 5G. Several fake theories allege that human exposure to radiations from 5G BSs can spread the Covid19 (Cellan-Jones, 2020; Temperton, 2020). Some examples of those fake theories are as follows.

- Cities that have started the deployment of 5G networks, e.g., Wuhan and Milan, have a wider spread of the virus compared to those employing legacy networks (Dailystar, 2020);

- The RFR from 5G severely interacts with the DeoxyriboNucleic Acid (DNA) of the cells in the lungs, leading to a deadly inflammation of lungs;

- The RFR from 5G weaken our immunity system, allowing the Covid19 to attack our bodies easily (Temperton, 2020);

- The Covid19 is a cover to embed microchips within the coronavirus vaccine to control humans via 5G (Goodman and Carmichael, 2020).

The fake theories and hoaxes reported above do not rely on any scientific evidence, and most of them are biologically impossible. For example, Covid19 has spread worldwide, mostly in cities that have not deployed $5 \mathrm{G}$ networks yet. Also, there is no proven interaction between the RFR and DNA of the cells. Moreover, $5 \mathrm{G}$ radiations do not affect the immune system because the radiation level is below the heating thresholds, defined by the well-established guidelines (Tuschl et al., 2006).

\subsection{Misconception of 5G Principal Concepts}

In this part, we investigate the potential health impacts of $5 \mathrm{G}$ from a communication theory viewpoint. We focus on the novel features in $5 \mathrm{G}$ that trigger health concerns among the community, i.e., massive multiple-input multiple-output (MIMO) and network densification.

\subsubsection{Massive MIMO}

In 5G, the BS adopts MIMO and beamforming techniques, which have also been considered in $4 \mathrm{G}$ networks. However, there are two substantial differences compared to previous networks, i.e., higher maximum output power and dynamic pencil beamforming with a larger number of antenna elements. The maximum transmitted power by a $5 \mathrm{G}$ BS can reach up to $200 \mathrm{~W}$, almost double the corresponding value for a $4 \mathrm{G}$ BS (Ericsson, 2018, 2013). This increase in power can trigger the population's concern about potential health risks.

Before judging the EMF exposure, we recall that the radiation pattern of BS is different from the previous generation, where it is more dynamic and adaptable to the spatial and temporal traffic distribution. On the contrary, the radiation pattern of MIMO $4 \mathrm{G}$ BSs is almost static with fixed beams over the area. Since the radiation pattern with massive MIMO varies over time and space, traditional assessment of compliance procedures to quantify the exposure can be misleading. These classical methods rely on conservative assumptions, e.g., all the users are in the same location that coincides with the testing point. These assumptions overly estimate the exposure from 5G BSs, leading to a lower maximum allowable power and a larger exclusion zone (Baracca et al., 2018; Chiaraviglio et al., 2020c).

To accurately quantify the exposure from $5 \mathrm{G}$ BSs, we should adopt statistical assessment of compliance procedures to account for the traffic and users' spatial distribution. In this regard, a statistical method to assess the exposure from 5G BSs is proposed in (Keller, 2019). Although the maximum radiated power in $5 \mathrm{G}$ is higher than that of $4 \mathrm{G}$, the radiation intensity is $25 \%$ of the maximum exposure for $95 \%$ of the cases. Also, the size of the exclusion zone is estimated to be less than half the value predicted by non-statistical methods (Thors et al., 2017). Therefore, we should consider statistical models to provide a realistic estimate of the exposure levels instead of the pessimistic conventional approaches that can wrongly trigger dispensable fair among the population.

\subsubsection{G Network Densification}

In order to support an increasing number of users with higher data rates, installing additional BSs over the territory is essential, i.e., network densification. Therefore, the distance between the users and the nearest cell shrinks, increasing the perceived health impacts.

Unlike the common opinion, network densification can significantly reduce the average EMF exposure for a given required rate (Chiaraviglio et al., 2020a; Chiaraviglio et al., 2019). In fact, the cell coverage area is smaller for networks with higher BS density, requiring lower transmitted power. On the contrary, networks with fewer BSs exhibit a larger coverage area and a higher radiated power for each cell. Furthermore, dense networks are endowed with a homogeneous distribution of power over the territory; hence, the EMF exposure is distributed more uniformly among the population. For networks with smaller densities, users close to BSs are exposed to higher radiation than those at the edge. In other words, network densification reduces both the average and the variance of the exposure over the area compared to legacy networks (Chiaraviglio et al., 2020a, Chiaraviglio et al., 2020d). 


\subsection{Open Questions}

One of the controversial aspects in the scientific community is the long-term non-thermal health impacts due to the exposure to non-ionizing RFR from cellular networks. The main reason is that there is not an established mechanism for inducing non-thermal severe health impacts. Nevertheless, some studies observe biological effects due to non-ionizing EMF exposure (Szmigielski et al., 1982; Chou et al., 1992; INTERPHONE Study Group, 2011; Larjavaara et al., 2011). In this regard, the International Agency on Research on Cancer (IARC) classified RFR as "Possibly carcinogenic to humans" (Group 2B) (IARC, 2011; IARC, 2013). Most of the studies investigating the biological impacts of RFR are animal-based experiments, where rats are exposed to RFRs to resemble the radiation from cellular networks. A massive number of animal-based experiments have been conducted to investigate the carcinogenic impacts of RFR (Wiktor-Jedrzejczak et al., 1980; Magras and Xenos, 1997; Lee et al., 2009; Hussein et al., 2016; Recordati et al., 2019; Yinhui et al., 2019). However, most of the studies have some methodological issues regarding the adequacy to investigate carcinogenic agents. For example, the duration of the studies, the number of animals, and the number of power intensity levels usually do not achieve the minimum requirements set in National Toxicology Program (NTP) and WHO to accurately judge the carcinogenicity of an agent (NTP, 2011; Gift et al., 2013). In the following, we investigate the potential health impacts of the exposure to RFR for frequencies below $6 \mathrm{GHz}$ and in the $\mathrm{mm}$ Wave band.

\subsubsection{Non-thermal Health Impacts of RFR Below $6 \mathrm{GHz}$}

Two recent studies by the NTP and Ramazzini Institute investigate potential biological impacts for the exposure to RFR with frequencies below $6 \mathrm{GHz}$. Both the experiments follow the adequacy guidelines in (National Toxicology Program, 2018a; National Toxicology Program, 2018b; Falcioni et al., 2018). In the NTP study, Sprague-Dawley rats were put in special chambers and exposed to RFR for several hours per day till nature death. The radio source generates modulated signals that mimic the signals produced by $2 \mathrm{G}$ and $3 \mathrm{G}$ networks. The considered center frequencies of the modulated signals are 900 and $1800 \mathrm{MHz}$ in (National Toxicology Program, 2018b) and (National Toxicology Program, 2018a), respectively. In (National Toxicology Program, 2018b), a malignant Schwannoma of the heart and a glioma of the brain were noticed in male rats exposed to high RFR intensity. Nevertheless, no statistically significant evidence for malignant tumors has been found in female rats in (National Toxicology Program, 2018b) and (National Toxicology Program, 2018a). Similarly, in (Falcioni et al., 2018), an increase in the number of male rats diagnosed with Schwannomas in the heart is noticed only for the highest EMF level.

Although the NTP and Ramazzini institute experiments follow the guidelines for investigating carcinogenicity (National Toxicology Program, 2018a; National Toxicology Program, 2018b; Falcioni et al., 2018), they have several issues in miming the radiation of $5 \mathrm{G}$ networks in realistic environments
(Chiaraviglio et al., 2020b). In particular, the animals are exposed to significantly higher power intensities compared to the exposure from a real BS and a user equipment (UE). Also, the signal format, exposure distance, average transmitted power per day, and maximum radiated power are not well configured to simulate the actual EMF exposure from 5G equipment.

In summary, more experiments are needed to investigate the biological effects, if any, of RFR from 5G. The studies should consider a sufficient number of animals and a duration longer than two years. Moreover, the RF sources should generate signal formats and modulation techniques adopted in 5G with maximum and average transmitted power that results in EMF exposure miming that of $5 \mathrm{G}$ BSs and UE.

\subsubsection{Communications in the Millimeter-Wave Band}

Some of the population is concerned that human exposure to EMF with higher frequencies such as $\mathrm{mm}$-Waves in $5 \mathrm{G}$ can have severe health impacts compared to lower frequencies, adopted in legacy networks. However, the penetration depth of mm-Waves inside the biological tissues is small, so the heating effect is mainly for the skin and eyes. In fact, the biological effects of mm-Waves have been investigated in several studies (Erwin and Hurt, 1981; Gandhi and Riazi, 1986; Riu et al., 1997; Kues et al., 1999; Ryan et al., 2000; Walters et al., 2000), albeit not in the context of 5G communications. The studies did not find adverse health impacts for $\mathrm{mm}$-Wave exposure with levels below the guidelines (WHO, 2020).

Nevertheless, since $5 \mathrm{G}$ BSs operating in $\mathrm{mm}$-Wave bands have not been widely deployed yet, more measurement studies and trials are needed to quantify the resulting exposure. Also, more bioassays should be conducted to investigate the non-thermal biological effects for the exposure to $\mathrm{mm}$-Waves with configurations, e.g., the power intensity and frequency, that simulate the radiation from a real BS and/or UE.

\section{PROPOSED EMF PRECAUTIONARY MEASURES}

As aforementioned, there are no proven adversary health impacts due to the exposure to the radiation from $5 \mathrm{G}$ BSs, provided that the RFR adheres to the exposure guidelines. However, EMFaware cellular system design is beneficial as a precautionary measure. In particular, cellular systems can be designed to preserve a given quality of service (QoS) in terms of the data rate and coverage while having minimal radiation. In this regard, two EMF-aware architectures for beyond $5 \mathrm{G}$ networks by exploiting emerging communication technologies are proposed. The first scheme considers the use of tethered unmanned aerial vehicles (UAVs), while the second exploits a reconfigurable intelligent surface (RIS) in smart environments.

\subsection{EMF-Aware Design With Tethered UAVs}

The exposure to EMF is usually dominated by the uplink (UL) exposure, as the UE is close to the users (Chiaraviglio et al., 2020b). Hence, one possible solution to reduce the UL exposure is considering green antennas, which operate in receiving-only 


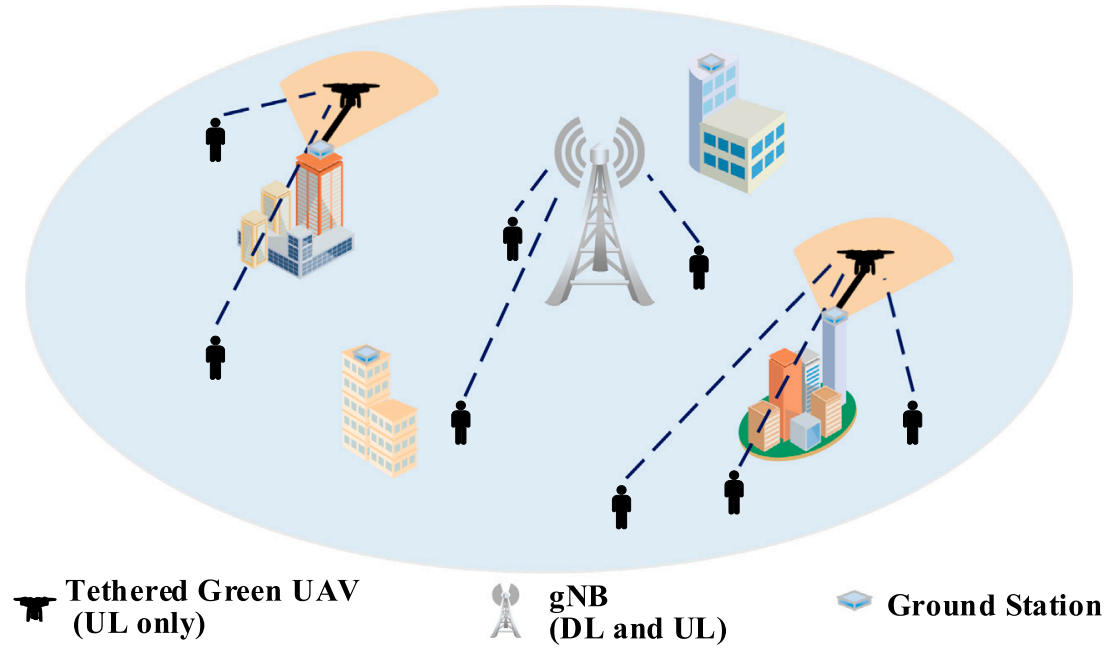

FIGURE 1 | The proposed EMF-aware cellular system design using tethered green UAVS for beyond 5 G networks.

mode. In this case, since the antennas are close to the user, the power transmitted from the UE and the UL exposure can be decreased. This is achieved by decoupling the downlink (DL) and UL, where all DL can be served by the macro cell BSs, while UL is fulfilled through green small cells (Ezri and Shilo, 2009). One possible issue is that once the green antennas are installed, they are fixed. Hence, these small cells cannot adapt to different users' spatial distribution and traffic. UAVs carrying small cells can offer a solution for the non-flexible deployment of green antennas. Nevertheless, there are some possible issues associated with UAVs: 1) they have limited battery capacity, limiting the flight time, payload, and number of serving users; 2) they need a wireless backhaul, which can increase the EMF exposure; 3) there are safety and privacy concerns for the free navigation of UAVs, especially in restricted areas near airports.

In this regard, an architecture is proposed in (Lou et al., 2021) to alleviate some of the problems associated with traditional UAVs, mainly the limited flight time and high power consumption. Regarding the flight time, tethered UAVs that can be connected to a pre-installed ground station at the top of some buildings are proposed, as shown in Figure 1. The tethering cable provides both power and data required for the UAV operation and their backhauling with the core cellular network, respectively. The proposed scheme can simultaneously solve limited battery capacity, safety and privacy concerns, and escalation of exposure due to wireless backhauling via tethering the UAVs. Also, green (i.e., receiving only) antennas are considered for the UAVs to support users' UL. Therefore, only the RF chain elements responsible for the UL are kept, reducing the power consumption. For instance, the RF chain does not necessitate having high power amplifiers, which are usually adopted for the DL to deliver a high amount of power. Those amplifiers have low power efficiency and consume considerable power, especially for higher frequency bands such as mm-Waves. For the EMF exposure and power consumption of the UE, the UAV locations are selected among the possible rooftops to minimize the exposure, and consequently the users' transmitted power along with their power consumption, while preserving a minimum target data rate for the users. Also, the tethered length and position of UAVs within their hovering area over the buildings can be optimized to minimize the exposure. The proposed scheme allows reducing the exposure by more than $50 \%$ compared to legacy networks with only macro cells while achieving a target data rate for the users, as shown in the initial results obtained in (Lou et al., 2021).

\subsection{EMF-Aware Design in Smart Environments}

In order to support a high data rate, the adoption of mm-Wave communication is vital. Nevertheless, the Line-of-Sight (LoS) path can be easily obstructed by blockages for mm-Waves, leading to severe performance deterioration. Hence, the UE has to increase its transmission power to cope with the path loss, escalating their EMF exposure. A possible solution to this problem is considering a relay to divide the link into two LoS propagation channels. The first is between the BS and relay, while the second is from the relay to UE. Nevertheless, relays are active devices with RFR that can increase the EMF exposure. On the other hand, RISs themselves are semi-passive elements, as they do not have RF chains, but work as reflectors, reducing the power consumption. The only power needed for them is just to run the circuits that control the phase, which is negligible compared to the power needed for transmission, as in relays.

The EMF exposure can be minimized by exploiting a passive controllable scatterer, i.e., RIS (Ibraiwish et al., 2021), as in Figure 2. RISs are reflecting surfaces that can improve the wireless channel by focusing the signal toward the receiver to establish a LoS link for the UE, especially when the direct LoS paths between the devices and BS are blocked. In this regard, RISs have been considered to maximize the communication and localization performance, e.g., the rate, coverage, and energy 


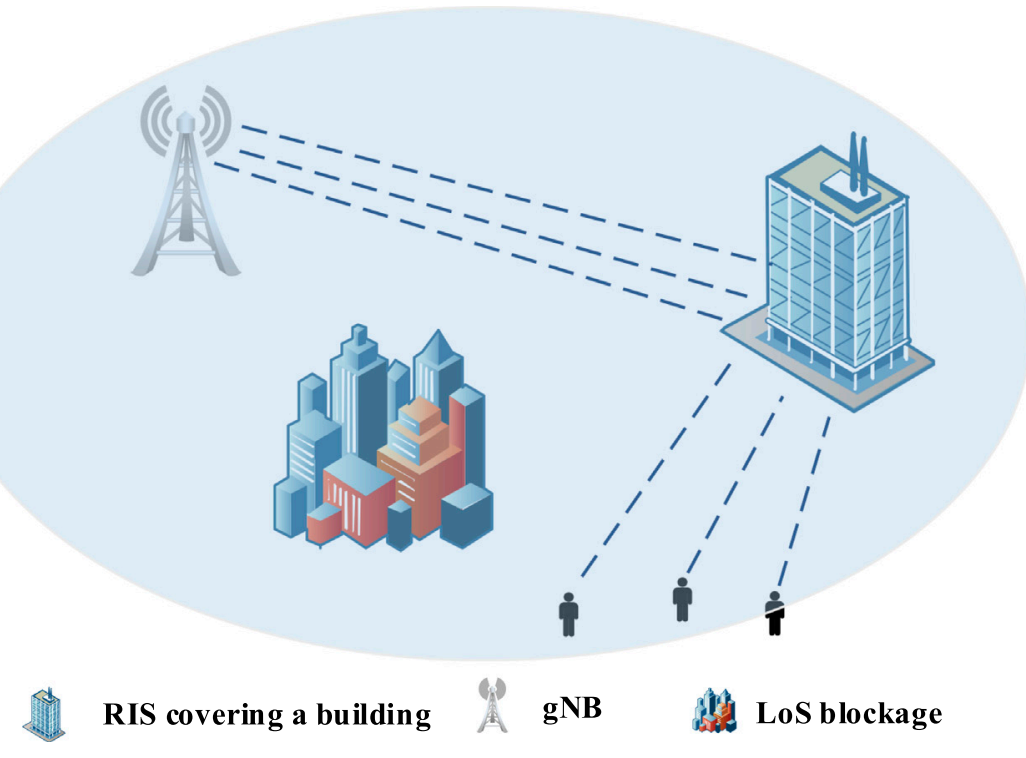

FIGURE 2 | The proposed EMF-aware cellular system design using a RIS for beyond 5G networks.

efficiency (Basar et al., 2019; Huang et al., 2019; Renzo et al., 2019; Elzanaty et al., 2020; Kishk and Alouini, 2020). In the proposed scheme in (Ibraiwish et al., 2021), the RIS phase profile and the beamformer at the BS are designed to minimize the population exposure to EMF quantified by an exposure index. The exposure index is a function of the transmitted power from the device and specific absorption rate (SAR) reference per unit transmission power, which depends on how the user grabs the UE among other factors. The scheme also necessitates achieving a target QoS in terms of required data rates for the users. The proposed architecture with optimized RIS phases can significantly reduce the users' exposure, transmitted power, and power consumption. For instance, compared to schemes without RISs, a $60 \%$ reduction in the exposure index and a $30 \%$ increase in the data rate can be achieved, as shown in the initial work (Ibraiwish et al., 2021).

\section{CONCLUSION}

The fair from $5 \mathrm{G}$ among the population is mainly due to fake theories and misconceptions of novel 5G features. There are no scientifically proven adverse health impacts from the exposure to RFR with levels below those suggested by the guidelines. Nevertheless, more research

\section{REFERENCES}

Baracca, P., Weber, A., Wild, T., and Grangeat, C. (2018). "A statistical approach for RF exposure compliance boundary assessment in massive MIMO systems," in Proc. 22nd international ITG workshop on smart antennas (WSA 2018). Bochum, Germany: VDE-VERLAG Publishing House.

Basar, E., Di Renzo, M., De Rosny, J., Debbah, M., Alouini, M.-S., and Zhang, R. (2019). Wireless communications through reconfigurable intelligent is required on long-term non-thermal impacts for EMF exposure, especially for mm-Waves. The experiments should consider RF sources that mimic the radiation from BSs and UEs with realistic signal formats, exposure distance, carrier frequency, and radiation power. As a precautionary measure, EMF-aware architectures for cellular systems can be considered to minimize the RFR. In this regard, emerging techniques in communications for beyond $5 \mathrm{G}$ systems, e.g., UAVs and RISs, can reduce the EMF exposure and increase the QoS.

\section{DATA AVAILABILITY STATEMENT}

The original contributions presented in the study are included in the article/Supplementary Material, further inquiries can be directed to the corresponding author.

\section{AUTHOR CONTRIBUTIONS}

The work was developed as a collaboration among all authors. MA, LC conceived the work. The manuscript was mainly drafted by $\mathrm{AE}$ and was revised and corrected by all co-authors. All authors have read and approved the final manuscript.

surfaces. IEEE Access 7, 116753-116773. doi:10.1109/ACCESS.2019. 2935192

BBC (2020). Coronavirus: derby $5 \mathrm{G}$ phone mast set on fire. Available at: https:// www.bbc.com/news/uk-england-derbyshire-52790399 (Accessed November 9, 2020).

Cellan-Jones, R. (2020). Coronavirus: fake news is spreading fast. Available at: https:// www.bbc.com/news/technology-51646309 (Accessed November 29, 2020).

Chiaraviglio, L., Bianchi, G., Blefari-Melazzi, N., and Fiore, M. (2020a). "Will the proliferation of $5 \mathrm{G}$ base stations increase the radio-frequency 'pollution'?, in 
Proc. IEEE Vehicular Technology Conference (VTC-Spring), Antwerp, Belgium, May 25-28, 2020. doi:10.1109/vtc2020-spring48590.2020.9128888

Chiaraviglio, L., Elzanaty, A., and Alouini, M.-S. (2020b). Health risks associated with 5G exposure: a view from the communications engineering perspective. Preprint arXiv:2006.00944.

Chiaraviglio, L., Rossetti, S., Saida, S., and Bartoletti, S. (2020c). Pencil beamforming increases human exposure to electromagnetic fields: true or false? Preprint arXiv:2010.16288

Chiaraviglio, L., Turco, S., Bianchi, G., and Melazzi, N. B. (2020d). "5G densification increases human exposure to radio-frequency pollution": true or false? Preprint arXiv:2010.00933.

Chiaraviglio, L., Fiore, M., and Rossi, E. (2019). "5G technology: which risks from the health perspective?," in The 5G Italy book 2019: a multiperspective view of 5G. 1st edn, Editors M. Ajmone Marsan, N. Blefari Melazzi, and S. Buzzi Consorzio Nazionale Interuniversitario per le Telecomunicazioni (CNIT), Italy Vol. 1.

Chou, C.-K., Guy, A. W., Kunz, L. L., Johnson, R. B., Crowley, J. J., and Krupp, J. H. (1992). Long-term, low-level microwave irradiation of rats. Bioelectromagnetics 13, 469-496. doi:10.1002/bem.2250130605

Dailystar (2020). Coronavirus: Activists in bizarre claim 5G could be acting as "accelerator" for disease. Available at: https:/www.dailystar.co.uk/news/weirdnews/coronavirus-fears-5g-wifi-networks-21728189 (Accessed November 29, 2020).

Elzanaty, A., Guerra, A., Guidi, F., and Alouini, M.-S. (2020). Reconfigurable intelligent surfaces for localization: position and orientation error bounds. arXiv preprint arXiv:2009.02818.

Ericsson (2013). Base Stations and networks. Tech. Rep., Ericsson. Available at https://tinyurl.com/y69eb2vf (Accessed April 21, 2020).

Ericsson (2018). EMF test report: Ericsson AIR 5121. Tech. Rep. Ericsson. Available at https://fccid.io/TA8AKRD901059-1/RF-Exposure-Info/RFExposure-information-3845517.pdf (Accessed March 24, 2020).

Erwin, D. N., and Hurt, W. D. (1981). Assessment of possible hazards associated with applications of millimeter-wave systems. Available at: https://apps.dtic. $\mathrm{mil} / \mathrm{dtic} / \mathrm{tr} / \mathrm{fulltext} / \mathrm{u} 2 / \mathrm{a} 112014 . p d f$ (Accessed May 27, 2020).

Ezri, D., and Shilo, S. (2009). "Green cellular - optimizing the cellular network for minimal emission from mobile stations," in IEEE International Conference on Microwaves, Communications, Antennas and Electronics Systems, Tel Aviv, Israel, November 9-11, 2009. 1-5. doi:10.1109/COMCAS.2009.5385989

Falcioni, L., Bua, L., Tibaldi, E., Lauriola, M., De Angelis, L., Gnudi, F., et al. (2018). Report of final results regarding brain and heart tumors in Sprague-Dawley rats exposed from prenatal life until natural death to mobile phone radiofrequency field representative of a $1.8 \mathrm{GHz}$ GSM base station environmental emission. Environ. Res. 165, 496-503. doi:10.1016/j.envres.2018.01.037

Foster, K. R., Ziskin, M. C., and Balzano, Q. (2017). Thermal modeling for the next generation of radiofrequency exposure limits. Health Phys. 113, 41-53. doi:10. 1097/hp.0000000000000671

Gandhi, O. P., and Riazi, A. (1986). Absorption of millimeter waves by human beings and its biological implications. IEEE Trans. Microwave Theor. Techn. 34, 228-235. doi:10.1109/tmtt.1986.1133316

Gift, J. S., Caldwell, J. C., Jinot, J., Evans, M. V., Cote, I., and Vandenberg, J. J. (2013). Scientific considerations for evaluating cancer bioassays conducted by the Ramazzini institute. Environ. Health Perspect. 121, 1253-1263. doi:10.1289/ ehp.1306661

Goodman, J., and Carmichael, F. (2020). Coronavirus: 5G and microchip conspiracies around the world. Available at https://www.bbc.com/news/ 53191523 (Accessed November 8, 2020).

Huang, C., Zappone, A., Alexandropoulos, G. C., Debbah, M., and Yuen, C. (2019). Reconfigurable intelligent surfaces for energy efficiency in wireless communication. IEEE Trans. Wireless Commun. 18, 4157-4170. doi:10. $1109 /$ twc.2019.2922609

Hussein, S., El-Saba, A.-A., and Galal, M. K. (2016). Biochemical and histological studies on adverse effects of mobile phone radiation on rat's brain. J. Chem. Neuroanat. 78, 10-19. doi:10.1016/j.jchemneu.2016.07.009

IARC (2011). IARC classifies radiofrequency electromagnetic fields as possibly carcinogenic to humans. Press release 208International Agency for Research on Cancer and others. Lyon, France: IARC. . Available at: https://www.iarc.fr/ wp-content/uploads/2018/07/pr208_E.pdf (Accessed November 29, 2020).

IARC (2013). Non-ionizing radiation, Part 2: radiofrequency electromagnetic fields. IARC Monogr. Eval. Carcinog. Risks Hum. 102, 1-460.
Ibraiwish, H., Elzanaty, A., Al-Badarneh, Y. H., and Alouini, M.-S. (2021). EMFaware cellular networks in RIS-assisted environments. Available at: http://hdl. handle.net/10754/666963.

ICNIRP (1998). Guidelines for limiting exposure to time-varying electric, magnetic, and electromagnetic fields (up to $300 \mathrm{GHz}$ ). International Commission on Non-Ionizing Radiation Protection. Health Phys. 74, 494-522.

ICNIRP (2020). ICNIRP guidelines on limiting exposure to time-varying electric, magnetic and electromagnetic fields $(100 \mathrm{kHz}$ to $300 \mathrm{GHz})$. Health Phys. 118, 483-524. Avaiable at: https://www.icnirp.org/cms/upload/ publications/ICNIRPrfgdl2020.pdf.

IEEE (2005). C95.1-2005 IEEE standard for safety levels with respect to human exposure to radio frequency electromagnetic fields, $3 \mathrm{kHz}$ to $300 \mathrm{GHz}$. Tech. Rep. New York, NY, US: Institute of Electrical and Electronics Engineers (IEEE).

INTERPHONE Study Group (2011). Acoustic neuroma risk in relation to mobile telephone use: results of the INTERPHONE international case-control study. Cancer Epidemiol. 35, 453-464. doi:10.1016/j.canep.2011.05.012

Jones, S. (2020). Switzerland halts rollout of $5 \mathrm{G}$ over health concerns. Available at: https://www.ft.com/content/848c5b44-4d7a-11ea-95a043d18ec715f5 (Accessed November 29, 2020).

Keller, H. (2019). On the assessment of human exposure to electromagnetic fields transmitted by 5G NR base stations. Health Phys. 117, 541-545. doi:10.1097/hp. 0000000000001089

Kishk, M. A., and Alouini, M.-S. (2020). Exploiting randomly-located blockages for large-scale deployment of intelligent surfaces, in IEEE J. Selected Areas Commun. 39 (4), 1043-1056. doi:10.1109/JSAC.2020.3018808

Kues, H. A., D’Anna, S. A., Osiander, R., Green, W. R., and Monahan, J. C. (1999). Absence of ocular effects after either single or repeated exposure to $10 \mathrm{~mW} / \mathrm{cm}^{2}$ from a $60 \mathrm{GHz} \mathrm{CW}$ source. Bioelectromagnetics 20, 463-473. doi:10.1002/(sici) 1521-186x(199912)20:8<463::aid-bem1>3.0.co;2-t

Larjavaara, S., Schüz, J., Swerdlow, A., Feychting, M., Johansen, C., Lagorio, S., et al. (2011). Location of gliomas in relation to mobile telephone use: a case-case and case-specular analysis. Am. J. Epidemiol. 174, 2-11. doi:10.1093/aje/kwr071

Lee, H.-J., Lee, J.-S., Pack, J.-K., Choi, H.-D., Kim, N., Kim, S.-H., et al. (2009). Lack of teratogenicity after combined exposure of pregnant mice to CDMA and WCDMA radiofrequency electromagnetic fields. Radiat. Res. 172, 648-652. doi:10.1667/RR1771.1

Liverpoolecho (2020). 5G mast "on fire" hours after mayor slams "bizarre" coronavirus conspiracy theories. Available at: https://www.liverpoolecho.co.uk/news/liverpoolnews/5g-mast-on-fire-hours-18041768 (Accessed November 09, 2020).

Lou, Z., Elzanaty, A., and Alouini, M.-S. (2021). Green tethered UAVs for EMFaware cellular networks. Available at: https://tinyurl.com/yxf8q9km.

Magras, I. N., and Xenos, T. D. (1997). RF radiation-induced changes in the prenatal development of mice. Bioelectromagnetics 18, 455-461. doi:10.1002/ (sici) 1521-186x(1997)18:6<455::aid-bem8>3.0.co;2-1

National Toxicology Program (2018a). Toxicology and carcinogenesis studies in B6C3F $1 / \mathrm{N}$ mice exposed to whole-body radio frequency radiation at a frequency $(1,900 \mathrm{MHz})$ and modulations (GSM and CDMA) used by cell phones. NTP Tech. Rep. Ser., NTP-TR-596. doi:10.22427/NTP-TR-596

National Toxicology Program (2018b). Toxicology and carcinogenesis studies in Hsd: Sprague dawley SD rats exposed to whole-body radio frequency radiation at a frequency $(900 \mathrm{MHz})$ and modulations (GSM and CDMA) used by cell phones. Triangle Park, NC: NTP Technical Report.

NTP (2011). Specifications for the conduct of studies to evaluate the toxic and carcinogenic potential of chemical, biological, and physical agents in laboratory animals for the national toxicology program.

Nyberg, R., and Hardel, L. (2017). 5G appeal: scientists and doctors warn of potential serious health effects of 5G. Available at: https:/www.jrseco.com/wpcontent/uploads/2017-09-13-Scientist-Appeal-5G-Moratorium.pdf. 5G appeal submitted to the European union (Accessed November 29, 2020).

Recordati, C., De Maglie, M., Marsella, G., Milite, G., Rigamonti, A., Paltrinieri, S., et al. (2019). Long-term study on the effects of housing C57BL/6NCrl mice in cages equipped with wireless technology generating extremely low-intensity electromagnetic fields. Toxicol. Pathol. 47, 598-611. doi:10.1177/ 0192623319852353

Renzo, M. D., Ntontin, K., Song, J., Danufane, F. H., Qian, X., Lazarakis, F., et al. (2019). Reconfigurable intelligent surfaces vs. relaying: differences, similarities, and performance comparison. Available at https://arxiv.org/pdf/1908.08747 (Accessed May 27, 2020). 
Riu, P. J., Foster, K. R., Blick, D. W., and Adair, E. R. (1997). A thermal model for human thresholds of microwave-evoked warmth sensations. Bioelectromagnetics $\quad 18, \quad 578-583$. doi:10.1002/(sici)1521-186x(1997)18: $8<578$ ::aid-bem6>3.0.co;2-\#

Robert Cleveland, F. J. L. U., and Sylvar, David. M. (1997). Office of engineering technology (OET) bulletin 65. Tech. Rep. Ed. 97-01. Federal Communications Commission (FCC).

Ryan, K. L., D'Andrea, J. A., Jauchem, J. R., and Mason, P. A. (2000). Radio frequency radiation of millimeter wave length: potential occupational safety issues relating to surface heating. Health Phys. 78, 170-181. doi:10.1097/ 00004032-200002000-00006

Szmigielski, S., Szudzinski, A., Pietraszek, A., Bielec, M., Janiak, M., and Wrembel, J. K. (1982). Accelerated development of spontaneous and benzopyreneinduced skin cancer in mice exposed to $2450-\mathrm{MHz}$ microwave radiation. Bioelectromagnetics 3, 179-191. doi:10.1002/bem.2250030202

Temperton, J. (2020). The rise and spread of a $5 \mathrm{G}$ coronavirus conspiracy theory. Available at: https://www.wired.com/story/the-rise-and-spread-of-a-5gcoronavirus-conspiracy-theory/ (Accessed November 08, 2020).

Thors, B., Furuskär, A., Colombi, D., and Törnevik, C. (2017). Time-averaged realistic maximum power levels for the assessment of radio frequency exposure for 5G radio base stations using massive MIMO. IEEE Access 5, 19711-19719. doi:10.1109/access.2017.2753459

Tuschl, H., Novak, W., and Molla-Djafari, H. (2006). In vitro effects of GSM modulated radiofrequency fields on human immune cells. Bioelectromagnetics 27, 188-196. doi:10.1002/bem.20191

Walters, T. J., Blick, D. W., Johnson, L. R., Adair, E. R., and Foster, K. R. (2000). Heating and pain sensation produced in human skin by millimeter waves: comparison to a simple thermal model. Health Phys. 78, 259-267. doi:10.1097/ 00004032-200003000-00003

WHO (2020a). Managing the COVID-19 infodemic: promoting healthy behaviours and mitigating the harm from misinformation and disinformation. Available at: https://tinyurl.com/y5crrd22 (Accessed November 29, 2020).

WHO (2020b). Radiation: 5G mobile networks and health. Available at https:// www.who.int/news-room/q-a-detail/5g-mobile-networks-and-health (Accessed November 15, 2020).

Wiktor-Jedrzejczak, W., Ahmed, A., Czerski, P., Leach, W. M., and Sell, K. W. (1980). Effect of microwaves $(2450-\mathrm{MHz})$ on the immune system in mice: studies of nucleic acid and protein synthesis. Bioelectromagnetics 1, 161-170. doi:10.1002/bem.2250010206

Yinhui, P., Hui, G., Lin, L., Xin, A., and Qinyou, T. (2019). Effect of cell phone radiation on neutrophil of mice. Int. J. Radiat. Biol. 95, 1178-1184. doi:10.1080/ 09553002.2019 .1607605

Conflict of Interest: The authors declare that the research was conducted in the absence of any commercial or financial relationships that could be construed as a potential conflict of interest.

Copyright (C) 2021 Elzanaty, Chiaraviglio and Alouini. This is an open-access article distributed under the terms of the Creative Commons Attribution License (CC BY). The use, distribution or reproduction in other forums is permitted, provided the original author(s) and the copyright owner(s) are credited and that the original publication in this journal is cited, in accordance with accepted academic practice. No use, distribution or reproduction is permitted which does not comply with these terms. 